\title{
Das Verhalten der Epiphysenlinie bei der Coxa Vara.
}

\author{
Von \\ Dr. Hermann Meyer-Göttingen, \\ Assistent der Kalinik.
}

Mit 13 Abbildungen in 'l'ext.

(Eingegangen am \&. Mai 1920.)

Literatur.

Brandes, Ubber Spätdeformation bei reponierten kongeritalen Hiiftgelenksluxationen und ihr Yerhältnis zum Krankheitsbilde der Osteochordritis deformans juvenilis. Zeitschr. f. orthop. ('hir. 35, Heft 2. 274. 1915. - v. Brunn, M., Chirurgische Krankheiten der unteren Extremitäten. Deutsche Chirurgie. Lieferung 66, II. Hälfte. - Drehmann, Coxa vara. Ergebn. d. Chir. u. Orthop. 2, 452. 1910. - Frangenheim, Zur Pathologic der Osteo-Arthritis deformans juvenilis des Hüftgelenks, über Coxa vara usw. Bruns' Beitr. 65, 19. 1909. - Derselbe, Weitere Untersuchungen über die Pathologie der Coxi vara adolescentium. Bruns' Beitr. 72, Heft 1. 239. - Derselbe. Die Krunkheiten des Knochensystems im Kirdesalter. Neue deutsche Chirurgie 10, 19l3. - Fromme, Über oine endemisch auftreter de Erkrankung des Knochensystems. Deutsche med. Woehenschr. 1919. Nr. 19. 510. - Derselbe, Die Ursache der Wachstumsdeformitiaten. Deutsehe med. Wochenschr. 1920, Nr. 7. 169. - Frösch, Zur Pathogenese đer Coxa vara. Mïnch. med. Wochenschr. 1919. Nr. 14. 375. - H aedke, Zur Ätiologie der Coxa vara. Deutsche Zeitschr. f. Chir. 66, 89. 1903. - Helbing, Coxa vara. Zeitschr. f. orthop. Chir. 15, 502. 1906. - HoeBly, Zur Frnge der Belastungsdeformitaiten. Münch. med. Wochenschr. 1919. Nr. 14. 373. - Hoff a, Die angeborene Coxa vara. Deutsehe med. Wochenschr. 1905. Nr. 32. 1257. - Hof moister. Schenkelhalsverbiegungen. Handb. d orthop. Chir. (Joachimsthal) घ, 365 1907. - Klopf er, Zur Frage der traumatischen Epiphysenlösungen. Bruns' Beitr. 89, Heft 2. - Landwehr, H., Über 3 Fälle von Solutio epiphyseos capitis fem. Coxa vara traumatica. Zentralbl. f. Chir. u. mech. Orthop. 6, Heft 1. l. - Lange, Lehrbuch der Orthopädie. Fischer, Jena 1914. - La a per, Zur Coxa vara. Deutsche Zeitschr. f. Chir. 116, 347. - Levy, Beiträge zur Frage der Coxitis, Coxa vara und sog. Osteoarthritis deformans juvenilis (besser Coxa vara cap.). Deutsche Zeitschr. f. Chir. 109, 205. 1911. - Lu B, Anatomische Beiträge zur Coxa varil. Diss. Wiurzburg 1899. M[anz, Die Ursachen der statischen Schenkelhalsverbiegung. Bruns' Beitr. 28, Heft 1. 29. - Peltesohn, Zur Ätiologje und Prognose der Coxa vara infantum. Zeitschr. f. orthop. ('hir, 28, Heft 3 u. 4. - Preiser, Georg, Coxa vara-Bildung nach Hüftluxation und allgemeine Hypoplasie von Knochenkerren. Zeitschr. f. orthop. Chir. 99, Heft 2. 115. Schmidt, Die Kontusion der Knorpelfuge des Schenkelkopfes und ihre Folgezustände. Mitteil. a. d. Grenzgeb. 1907. Mikulicz Gedenkband 77. - Schwarz, Über die Coxa vara congenita. Bruns' Beitr. 87, Heft 3. 685. 1913. - Derselbe, Zur Frage der spontanen Epiphysenlösung (intrakapsuliure Schenkelhalsfraktur?) im Kindesalter. Bruns' Beitr. 
87. Heft 3. 709. 1913. - Sudeck, Zur Anatomie und Ätiologie der (oxa vara adolescentium. Arch. f. klin. Chir. 59, Heft 2. 504. 1899. - Staffel, Beitrag zu unserer Kenntnis von den Trochanterdeformitäter. Zeitschr. f. orthop. Chir. 34, 538. 1914.

Bekamntlich spielt die Epiphysenfuge im Krankheitsbilde der Coxa vara eine große Rolle. Sowohl bei der Entstehung als auch als Unterscheidungsmerkmal der verschiedenen Arten hat man sie herangezogen und dabei ein bestimmtes gesetzmäßiges Verhalten, besonders bei den Hauptgruppen, der angeborenen, der rachitischen und der statischen Form feststellen können. Für die Coxa vara congenita, wie sie 1897 Hoffa zuerst beschrieb, ist eigentümlich der senkrechte Verlauf der Epiphysenlinie, dicht am Trochanter, so daß vom Hals kaum etwas vorhanden ist, im Gegensatz zu der Rachitis, bei der die Epiphysenlinie mehr schräg von außen oben nach innen unten verläuft, erheblich verbreitert und unscharf begrenzt ist. Und schließlich zieht sie bei der Statika schmal, meist zackig begrenzt wie ein großes Komma in einem nach außen oben offenen Bogen um den ebenfalls verkrïmmten Hals nach abwärts, den Kopf wie eine lang ausgezogene, schmale Mondsichel begrenzend. Derartige, dem Rōntgenbilde. entlehnte Eigentümlichkeiten können jedoch erst dann eine einwandfreie pathologisch-anatomische Deutung erfahren, wenn die Röntgenbefunde mit den gewonnenen Coxa vara-Präparaten eingehend verglichen werden. Dabei hat zwar die Wertung der Rọntgenbilder manche Wandlung durchgemacht; denn wir dürfen eines nicht vergessen: So wertvoll auch eine Rōntgenuntersuchung für die klinische Beurteilung gerade zur Abgrenzung der Coxa vara gegen Koxitis, kongenitale Luxation und Osteochondritis sein kann, so ist sie infolge der flächenhaften Projektion und ihrer Abhängigkeit vom Röhrenstand, von der Lagerung und von Dickenunterschieden nicht imstande, uns ein eindeutiges Bild von Verbiegungen des Halses, seinen Strukturverhältnissen und Verlagerungen des Kopfes zu geben. Damit dürfte auch die übertriebene Bewertung der Winkelmessung (Schenkelhalsneigungswinkel, Richtungswinkel Alsbergs und Epiphysenwinkel Helbings), einst das wichtigste an der ganzen Röntgenuntersuchung, in ihre Schranken zurückgewiesen sein. Erst in neuerer Zeit hat sich die Aufmerksamkeit immer mehr der Epiphysenfuge zugewendet in der an Hand von Präparaten gewonnemen richtigen Erkenntnis, daß in der weitaus größten Zahl der Coxa vara-Fälle der Krankheitsprozeß sich hier abspielt. Dieses Interesse hat zwar die Pathologie der Epiphysenfuge wesentlich gefördert und ihre Bedeutung in größerer Perspektive, nämlich für die Deformitäten überhaupt aufgedeckt. Aber wir sind noch weit davon entfernt, auf diesem Gebiete klar zu sehen. Ich erinnere bloß an die Deutung, welche die Coxa vara congenita von den verschiedenen Autoren erfahren hat. Während Haedke auf Grund histologischer Untersuchungen eine Rachitis annimmt, bezeichnet Hoffa das Fehlen jeden Wachstums als eigentümlich und führt dieses auf eine angeborene Störung in der Ossifikation entweder auf Grund einer fötalen Rachitis oder eines Keimfehlers zurück. Begründet sind diese Verhältnisse wohl in den Schwierigkeiten, welche sich dem Untersucher bei der experimentellen Bearbeitung der Wachstumsund Emährungsverhältnisse der Epiphysenfuge, sowie ihrer histologischen Untersuchung entgegenstellen. Aus diesem Grunde wird das Röntgenbild trotz 
seiner eingangs erwähnten Unzulänglichkeiten ein wertvolles Hilfsnittel auch: in der Beurteilung der Epiphysenlinie und der Kalksalzeinlagerungen bleiben.

Wie sieht mun normalerweise eine Epiphysenfuge und ihre Umgebung auf dem Röntgenbilde aus? Am gesunden Fermur entspricht die Knorpelfuge einem mäßig gespannten lineären Bogen, der ohne Unterbrechungen $1 / 2-11 / 2 \mathrm{~mm}$ breit entweder fast horizontal oder leicht von oben außen nach innen unten geneigt verläuft. Die Konvexität des Bogens schaut dabei nach dem Gelenk zu. Ihm sitzt der Kopf, in Wirklichkeit fast kugelrund, auf dem Röntgenbilde in Form einer Orangenschnitte auf, den Hals oben und unten etwas überragend. Jedoch ist dieses Bild erst vom 6. Lebensjahre ab vorhanden. Bis dahin ist die Knorpelfuge vermöge ihrer Eigenschaft als Wachstumszone von Jahr zu Jahr recht eingehenden Yeränderungen unterworfen, die zwar auch nach dieser Zeit bis zum Abschluß des Wachstums anhalten, aber alsdann viel langsamer vor sich gehen und keine so große Bedeutung mehr für die Gestalt des koxalen. Femurendes besitzen.

Einige entwicklungsgeschichtliche Daten mögen dieses erhellen. Bekanntlich geht das Längenwachstum vorwiegend von der diaphysären Oberfläche der Epiphysenscheibe aus. Ihren Anteil gibt Poland (zitiert nach Klopfer) mit 14 Fünfzehntel der Gesamtlänge des Knochens an. Am Femur ist die untere Epiphysenfuge die größere und leistet für das Längenwachstum das doppelte der oberen. Die Funktion der letzteren ist dafür eine um so mannigfaltigere. Hängt doch ron ihr der sowohl statisch als auch anatomisch so schwierige Bau des oberen Femurendes mit seinem Hals und dessen Neigung zur Schenkelschaftachse und seiner Kopf- und Trochanteranlagerung ab. Die obere Knorpelknochengrenze verläuft im 4. Fötal-

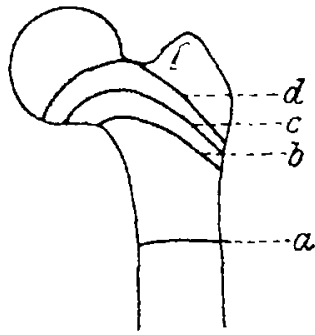

Abb. 1 . monat ganz geradlinig (Abb. 1). Sie hat beim Neugeborenen leicht bogenförmige Gestalt mit einer stärkeren hakenförmigen Krümmung nach dem in Bildung begriffenen Halse zu. Thr.Verlauf nähert sich nun immer mehr dem einer Parabel, so daß sie mit $41 / 2$ und 7 Jahren das durch die oberen Linien der Abb. 1 angedeutete Aussehen erhält. Somit nimmt der Hals erst im Laufe der ersten Le bensjahre seine endgültige Gestalt an. Ebenso wichtig fïr die Pathologie der Coxa vara wie ciese Tatsache ist auch das Auftreten der ersten Kalksalzeinlagerungen in der Kopfund Trochanterepiphyse und ihre Weiterentwicklung. Im Schenkelkopf entsteht ein Kern am Ende des ersten Lebensjahres, in der Trochanteranlage im 3.-4., im Trochanter minor erst im 12.-14. Jahre. Dieser verschmilzt zuerst mit dem Körper (im 17. Lebensjahre). Es folgen ihm der Trochanter major, dann der Kopf. Die Angaben der Autoren bewegen sich zwar in sehr weiten Grenzen. Wenn aber die innigen Beziehungen der Ossifikation zur Rachitis und zu den Drüsen mit innerer Sekretion bedacht werden, so könnte man geneigt sein, hierbei krankhafte Einflüsse anzunehmen.

Histologisch findet sich in der Knorpelfuge eine regelmäßige Anor dnurg der Knorpelzellen, die hauptsächlich nach dem Hals zu Reihenbildung erkennen lassen (Säulenknorpel). Kopfwärts rom Säulenknorpel liegt eine Schicht, die durch vermehrte Zellbildung sich vom rubenden Knorpel des Kopfes abhebt. Nach dem Halse zu folgt die Verkalkungs- 
zone, in welche die primären Markräume hincinwuchern. In iahnlicher Weise findet von ihrem. Knochenkern uus Wachstum und Verknöcherung dor Epiphyse statt.

Die Verkittung der Diaphyse mit der Epiphyse in der Knorpelwucherungs. zone ist gar nicht so fest. Wenr sich trotzdem experimentell durch Zug eine Epiphysenlösung schwierig herstellen läßt (Kirmisson), so hat dies seinen Grund darin, daß die zugfeste Knochenhaut beide Teile zusammenhält. Zwar haben mechanische Kräfte (Zug und Druck) nach den Experimenten von Maas einen bestimmenden Einfluß auf die Tätigkeit der Knorpelwucherungszone. Hoffmeister ist sogar geneigt, in den Maasschen Versuchen einen Beweis dafür zu erblicken, daß einseitiger Zug oder Druck auf die Epiphyse des wachsenden Knochens zur Änderung der Wachstumsrichtung, zur Verkrümmung führt. Bei einem ausgewachsenen Knochen ist aber, wenn nicht Frkrankungen des Knochens selbst vorliegen, die Annahme eines mechanischen Einflusses auf die Knochengestalt nicht mehr berechtigt, da sich die Knochenlamellen, gemäß ihrer funktionellen Inanspruchnahme, in einem ganz gesetzmäßigen und widerstandsfähigen Zug- und Druckbogensystem eingestellt haben. Mit der Epiphysenfuge steht dieses System am oberen Femurende insofern in Beziehung, als nach Sudeck die äußerste Lage der Zugbogenlamellen mit ihr zusammenfüllt. Gibt nun das Zugbogensystem nach - das Druckbogensystem ist bedeutend stärker angelegt - (Ada mscher Bogen), so kommt es zur Coxa vara-Bildung. An dieser am meisten beanspruchten Stelle findet sich daher nach Sudeck zur Verstärkung des zu schwachen Zugbogens regelmäßig eine

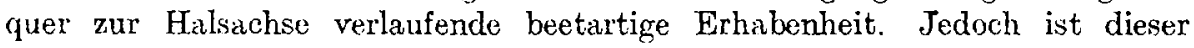
Beweis für die Sudecksche Coxa var'u-Theorie nicht ganz ïberzeugend, zumal ein solcher Höcker, welcher schon von Anatomen, wie Henle und Poirier, beschrieben worden war, sich an anderen Teilen des Knochenskeletts, die einer ahnlichen Beanspruchung auf Zug und Biegung unterliegen, nicht gefunden wird. Allerdings konnte R. Fick insofern Sudecks Untersuchungen bestätigen, als er diese, gratartige Erhebung" (Kocher) bei 80 normalen Oberschenkeln nur 7 mal nicht fand.

Wann sind wir denn überhaupt berechtigt, aı̣ Grund eines Röntgenbildes von einer Coxa vara zu sprechen? Bei der Varus-Stellung eines Gliedes greht bekanntlich sein körperferner Teil eine Annäherung an die Mittellinie des Körpers ein. Dieses kann am oberen Femurende sowohl durch eine Verschiebung des Kopfes gegen den Hals, eine Krümmung des Halses selhst, eine Achsenveränderung des Schaftes gegen den Hals, als auch durch eine Abknickung im Schaft unterhalb des großen Rollhïgels erfolgerr.

Wie sind diese Teile normal gegeneinander gelegen? Abb. 2 stellt diese Achsenverhältnisse schematisch dar. Der Schenkelhals bildet mit dem Schaft im Mittel einen Winkel von 125 bis 126 Grad nach Mikulicz. Während aber beim Schenkelhalswinkel individuelle und Altersschwankungen von kaum 5-10 Grad vorkommen, bewegen sich die anderen eingarrgs erwähnten Winkelmessungen in erheblich woiteren Grenzen. So ist der Richtungswinkel Alsbergs, der durch die Oberschenkelschaftachse und die Verbindungslinie der Knorpelendpunkte des Kopfes gebildet wird, im Durchschnitt mit 41 Grad ermittelt, weist aber normal als untersten Grenzwert 29 Grad, als höchsten 51 Grad auf. Der Epiphysenwinkel Helbings wird in ähnlicher Weise ge- 
messen, nur daß statt der Knorpelendpunkte die rōntgenologisch höchstens vermutet werden können, die geradlinig verlängerte Epiphysenfuge als Schenkel benutzt wird. Der Winkel e in Abb. 2 schwankt zwischen 39 Grad und 62 Grad und ist von Helbing durchschnittlich mit 57 Grad ermittelt. Weist handelt es sich aber schon um eine Coxa vara, wenn e kleiner als 57 Grad ist.

Abgesehen von diesen am Untersuchten gefundenen normalen Schwankungen erschweren die infolge des oft bogenförmigen Verlaufes vor Achsen und Epiphysenlinien unvermeidbaren Meßfehler des Untersuchers die Bewertung und den Vergleich derartiger Messungen erheblich. Nur im Rahmen des ganzen Krankheitsbildes unter Berücksichtigung der klinischen und der iibrigen röntgenologischen Erscheinungen lsönnen sie daher ein diagnostisches Hilfsmittel bedeuten. Das Verhalten der Epiphysenfuge bei den Hauptgruppen der Coxa vara ist za Anfang dieser Abhandlung kurz gestreift. Soweit sich die spärlichen Mitteilungen auf diesem Gebiete aber in Einzelheiten ergehen, sind sie Gegenstand lebhafter Meinungsverschiedenheiten geworden. Das gab mir Veranlassung, nicht allein diese Streitfragen, sondern auch die allgemein anerkannteu Merkmale der Coxa vara-Epiphyse an Hand eines reichhaltigen Materiales einer Nachprüfung zu unterziehen. Die vorwiegend röntgenologischen Studien erstrecken sich über 75 Fälle. Sie bilden eine Auswahl aus der weitaus größeren Zahl sämtlicher Schenkelhalsverbiegungen, welche getroffen wurde, weil nur technisch vollkonmene Bilder, die auch Finzelheitern erkennen lassen, den vorliegenden Zwecken genügen könmen. In der Besprechung

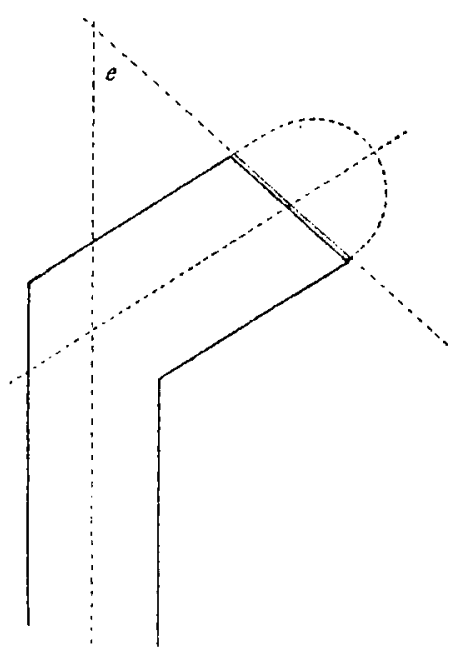

Abl. :2. der einzelnen Gruppen sei dit Helbingsche Einteilung der Coxa vara zugrunde gelegt.

Die Coxa vara kann auftreten:

A. Als angeborenes Leiden:

1. Für sich ohne sonstige Deformitïten.

2. Als congenitaler Oberschenkeldefekt.

3. In Verbindung mit anderen angeborenen Doformitäten:

a) Als Teilerscheinung einer Iuxatio coxae congenita.

b) In Verbindung mit angeborenen Deformititen anderer Gelenke.

B. Als postfötal erworbenes Leiden bei:

1. Rachitis.

2. Einer noch nicht sicher zu bestimmenden Erkrankung des Wachstumsaiters.

3. Osteomalazie.

4. Ostitis fibrosa.

5. Osteomyelitis. 
6. T'uberkulose.

7. Zystenbildung und malignen zystischen 'Tumoren.

8. Arthritis deformans.

9. Außeren Gewalteinwirkungen.

Die angeborene Coxa vara ist verhältrisniäbig selten. Ich fand sie nur 6 inal. Das Röntgenbild erscheint nach dem Literaturstudium auf den ersten Blick so typisch, daß die Diagnose scheinbar nach ihm allein gestellt werden kann. Der Schenkelkopf ist kleiner und hat sich in der Pfanne so gedreht, daß ex mit seinen unteren Teilen den Pfannenrand verlassen hat. Die Epiphysenlinie ist $2-3 \mathrm{~mm}$ breit, zackig begrenzt und zieht im oberen Teile senkrecht nach unten, um sich in der Mitte gabelförmig zu teilen. Dadurch erscheint ein dreieckiges, unregelmäßBig begrenztes Knochenstiick am unteren

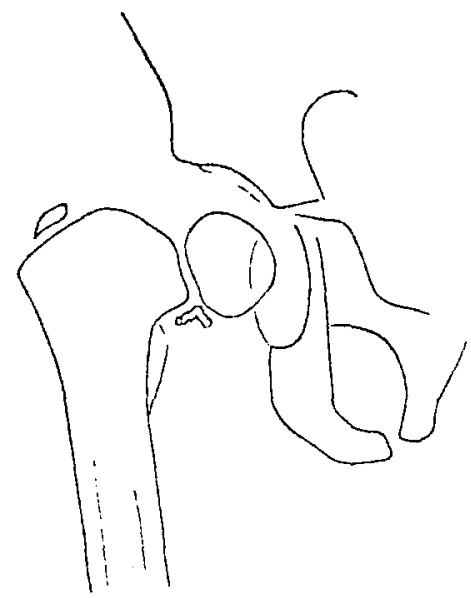

Hit 3 Jahren.

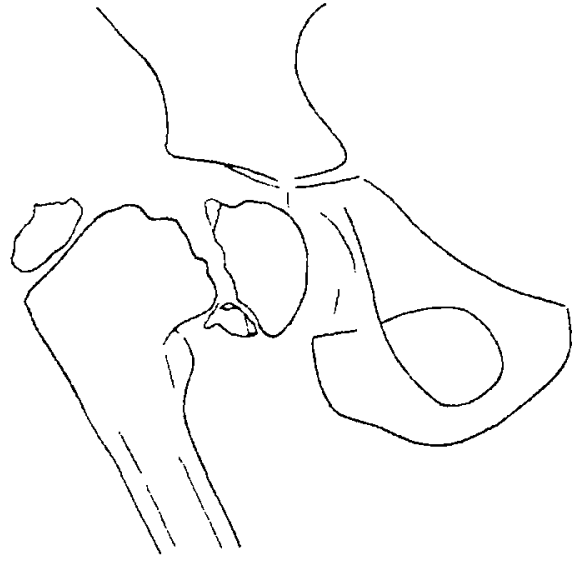

Mit 5 Jahien.

Abb. 3. Coxa vara congenita.

Schenkelhalsrande wie ausgesprengt und bat im Verein wit der bandartigen, abnorm verlaufenden Epiphysenlinie schon öfters Veranlassung zur Fehldiagnose: „Fraktur" gegeben. Die Epiphysenfuge grenzt dem Schenkelschaft unmittelbar an, so daß ein Schenkelhals gewöhnlich vollkommen fehlt. Ist aber ein solcher angedeutet, so ist er direkt an seinem Femuransatz mehr oder weniger rechtwinklig abgeknickt.

Abb. 3 möge diese Verhältnisso erläutern. Von einer Reproduktion der Original. platte ist abgesehen, da seine Feinheiten vollkommen verloren gehen wïrden und dururtige Abbildungen noch nicht einmal das Wesentliche wiederzugeben vermögen. Mir ist wenigstens in der Literatur eine einwandfreie Wiedergabe von Coxa vara-Röntgenbildern nicht zu Gesicht gekormen.

Der Schenkelhalswinkel bewegt sich, soweit er ïberhaupt meßbar ist, um 90 Grad, der Epiphysenwinkel zwischen $+20^{\circ}$ und $-20^{\circ}$. Außerdem findet sich nicht so selten eine Asymmetrie des Beckeneinganges. Der absteigende und der horizontale Schambeinast sind gegenüber der gesuuden Seite verschmälert und verkleinert. Ebenso erscheint das Foramen obturatorium enger. 
Das Auffallendste an diesem von Hoffa und Helbing so schirf umgrenzten und eingehend beschriebenen Röntgenbefunde ist der eigentümliche Verlauf der Epiphysenfuge oder des Gebildes, das als solche angesprochen wurde. Vergebens wird man bei beiden Autoren nach einer Erklärung für diese bandartige Aufhellung im Halse suchen, wenn nicht der histologische Befund für sich selbst sprechen soll. Makroskopisch ist jedenfalls eine Spaltbildung an dem Epiphysenverlauf nicht vorhanden, sondern es findet sich im Anschluß an die Epiphysenfuge nach dem Halse zu undifferenziertes, noch nicht verkalktes Knochengewebe. Hoffa hatte demgemäß auf Grund seiner Resektionspräparate die aufgehellte Zone als verbreiterte Epiphysenfuge aufgefaßt. Frst Schwarz macht an Hand von sehr klaren Röntgenbildern darauf aufnerkyam, daß in der bundartigen Aufhellung die Epiphysenfuge gar nicht verläuft, sondern als ganz feiner Spalt beckenwärts durch das große Kopf-

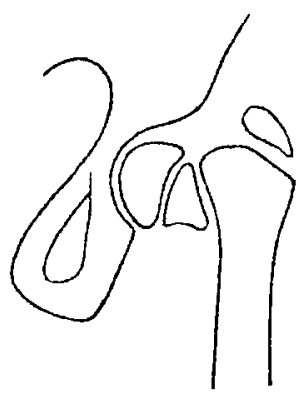

Abb. $4 a$. Nach Drehmann

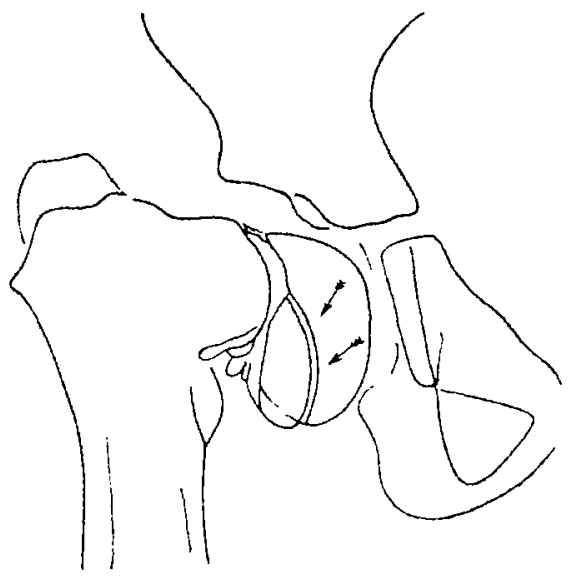

Abb. 4 b. Coxa vara congenita. 6 Jahre.

stück zieht, dieses in die eigentliche Epiphyse und ein Halsfragment teilend. So findet jedenfalls das am unteren Halsrande liegende dreieckige Sprengstïck eine hinreichende Erklärung. Das aufgehellte Band bezeichnet Schwarz als Schenkelhalsfuge, für die er undifferenziertes Knorpelgewebe annimmt, und welches infolgedessen bei der Belastung einen Locus minoris resistentiae abgeben muß. Die Verschiebung geht nicht etwa in der Epiphysenfuge, sondcrn in dieser Schenkelhalsfuge vor sich. Der Beweis für das Vorhandensein einer solchen Schenkelhalsfuge steht aber aus.

Daß nicht immer dieser bandartige helle Streifen mit der Epiphysenfuge zusammenfält, hatte schon D reh man $n$ beobachtet, der auf dem in $\mathrm{Abb}$. $4 \mathrm{a}$ wiedergegebenen Befunde seine Ansicht vom Zusammenhang der Coxa vara mit dem angeborenen Oberschenkeldefekt aufbaute. Das ausgesprengte Knochenstïck im unteren Halsteil nimmt die ganze Halshöhe ein. Auch ich fand ein Bild, das den von Schwarz und Drehmann aufgedeckten Verhältnissen sehr nahe kommt. Abb. $4 \mathrm{~b}$ gibt den Befund wieder. Man erkennt wohl deutlich neben dem hellen Bande einen bogenförmigen Spalt nach dem Kopf zu 
(Pfeil). Beide umgrenzen im unteren Halsteil ein Knochenstïck, das sich der Dreieckform nähert und gut die halbe Halshöhe einnimmt. Wenn man sich die Aufhellung fortdenkt, und Kopf und Hals in ihre Begrenzung in der Epiphyse zueinander ergänzt, so fallen die hinteren Umrisse mit dem hellen Bande zusammen, so daß man geneigt sein könnte, die ganze Erscheinung auf die infolge der Halsbiegung wach unten und hinten veränderte Projektion bei starken Kalkmangel im epiphysären Halsteil zurückzuführen. Wiederum ist bei anderen Bildern dieser Erklärungsversuch unmöglich. So bringen uns in Abb. 5 und 6 die Projektionsverhältnisse nicht weiter, wenngleich bei $\mathrm{Vi}$ eine schmale Epiphyserlinie angedeutet ist.

Vielmehr sieht es hier so aus, als ob die Knochenbildung halswärts schon sehr frïh eingestellt gewesen sei und nur am unteren Halsrand dem Druck-

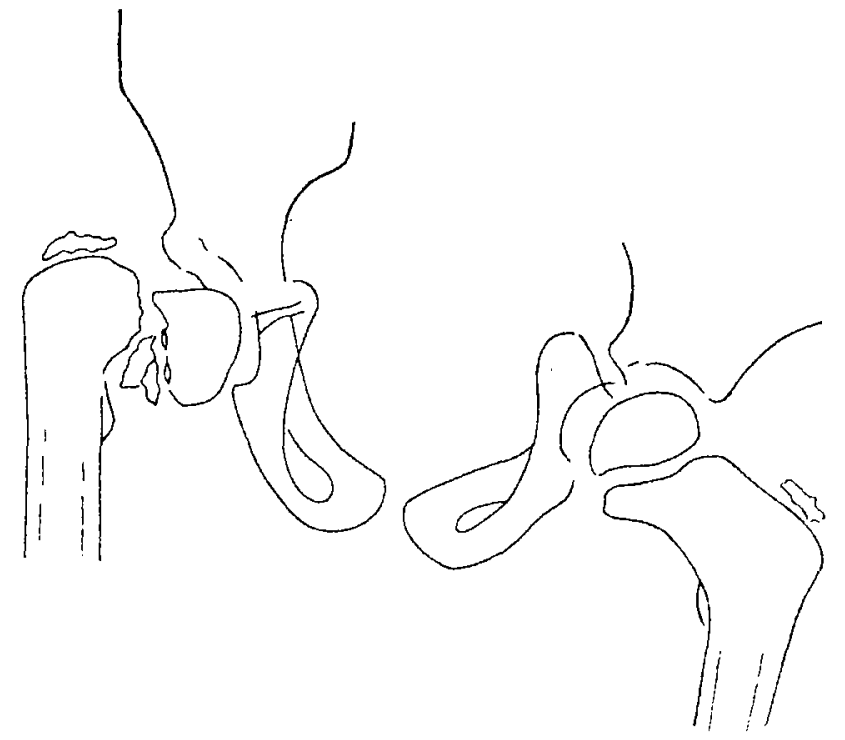

Abb. 5. Coxa vara congenitia. is Jahlire.

bogensystem entsprechend wohl als Folge der Belastung eine vermehrte Knochenbildung eingesetzt hätte. Der Kopf dagegen erscheint kaum verändert. Nul gegenuiber dem unteren Pfinnemrande hat er seine schöne Rundung verloren und sieht wie abgeschliffern aus. Auch die Trochanterepiphyse ist beachtenswert. Sie erscheint bedeutend kürzer und breiter und geht direkt in die Kopfepiphyse über.

Allerdings sind diese beiden Fälle dadurch kompliziert, daß klinisch Anzeichen einer überstandenen Rachitis vorhanden waren, wenn auch die Coxa vara schon im frühen Kindesalter beobachtet worden ist.

Rachitisverdächtig sind die anderen Schenkelhälse mit ihrer geschweiften und orheblich verbreiterten Epiphysenfuge und der spornartig ausgezogenen unteren Halsspitze.

Wenn sie demnach zur kongenitalen Coxa vara gezählt werden sollen, so behält die Erklärung sämtlicher. Erscheinungen ohne Berücksichtigung 
der Rachitis etwas Gezwungenes. Werden sie ganz als Rachitis aufgefaßt, so ist damit der Beweis erbracht, daß die bandartigen Aufhellungen im Schenkelhals auch bei ihr vorkommen kömnen, ähnlich wie sie von Fraenkel und Lorey bei ausheilender Allgemeinrachitis in der Tibia- und Femurmetaphyse beschrieben worden sind. Diese Fugen bestehen aus osteoidem Gewebe und verschwinden bis auf schmale Kalkstreifen (Jahresringe). Zwar ist das am Schenkelhalse nicht der Fall, die breite Fuge bleibt. Aber hat sich die Störung der Ossifikation fötal oder kurz nach der Geburt schleichend entwickelt oder ist sie gar in einer fehlerhaften Keimanlage begründet, so diß das osteoide Gewebe die Neigung, sich zu verknöchern, verloren hat, so müssen nach $A b$ heilung des Prozesses ähnliche Bilder wie bei der kongenitalen Coxa vara zustande kommen.

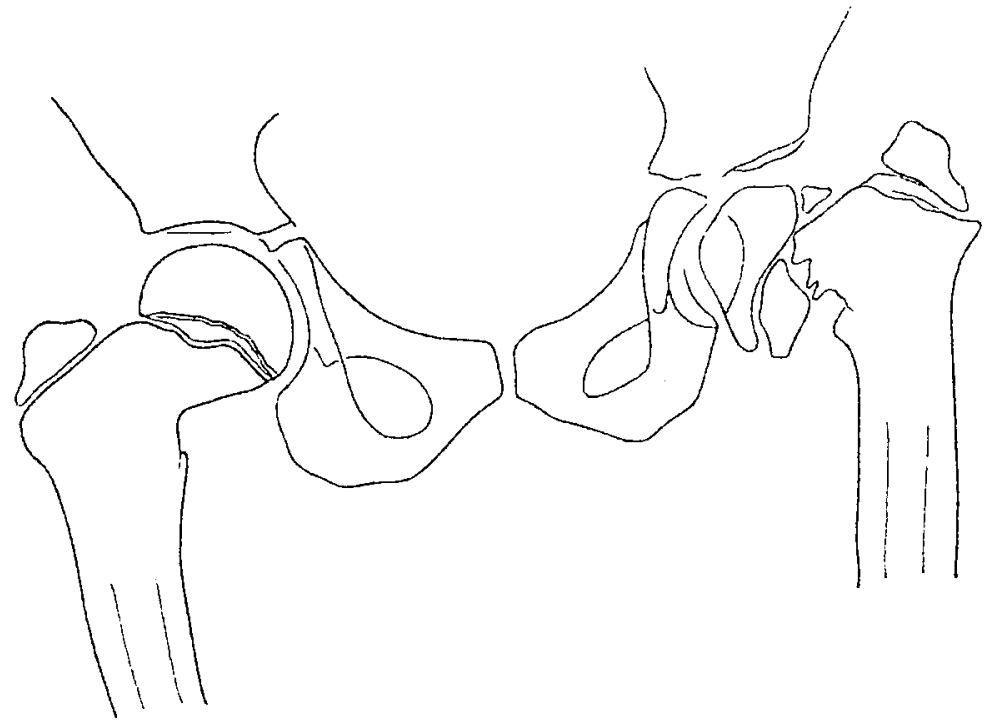

Abb. 6. Coxa vara congenita. 7 , Tahre.

Gerade diese Erscheinungen sind differentialdiagnostisch sehr wichtig, da in der Tat zwischen der Kongenita und der Rachitis Ubergänge beobachtet worden sind, wie auch Hoffa selbst hervorgehoben hat. Im Zweifelfsfalle müssen die Uberreste einer Rachitis für die Diagnose den Ausschlag geben. Im algemeinerr kann man aber als typischen Röntgenbefund für die Coxa vara congenita gelten lassen: den nahezu senkrechten Yerlauf der bandartigen Aufhellung, das Fehlen dos Halses und den großen Kopfteil. Soweit meßbar, schwankte in meinen Fällen der Epiphysenwinkel zwischen - 22 Grad und +25 Grad, der Schenkelhalswinkel zwischen 72 Grad und 87 Grad.

Nach reponierter angeborener Hüftluxation konnte ich 12 mal eine Coxa vara feststellen. Abgesehen von den Veränderungen, wolche sich bei Hüftluxationen am oberen Femurende recht oft finden, Knorpelwucherungen in der Pfanne, Deformierungen des Kopfes -(Pufferkopf), Halsverdickungen ist für die Coxa vara noch charakteristisch der schollige Zerfall des Kopfes oder 
sein vollkommener Schwund bis auf ein paar winzige Kalksalzflecken, so daß die Bilder schließlich der Osteochondritis (Perthes) erheblich ähnlich sehen (Abb. 7). Die Epiphysenfuge ist fast horizontal oder leicht von außen oben nach innen unten geneigt und verläuft meist bogenförmig. Thre Begrenzung ist aber häufig unscharf, wie zerzaust oder sie ist ganz unterbrochen. Zuweilen ist ein cleutlicher Kalksalzmangel in der Umgebung der Epiphyse erkennbar, während die ïbrigen Kopf- und Halsteile dichte Struktur zeigen. Am augenfäligsten bleibt aber der geradezu ïbertrieben plumpe Hals und Trochanter.
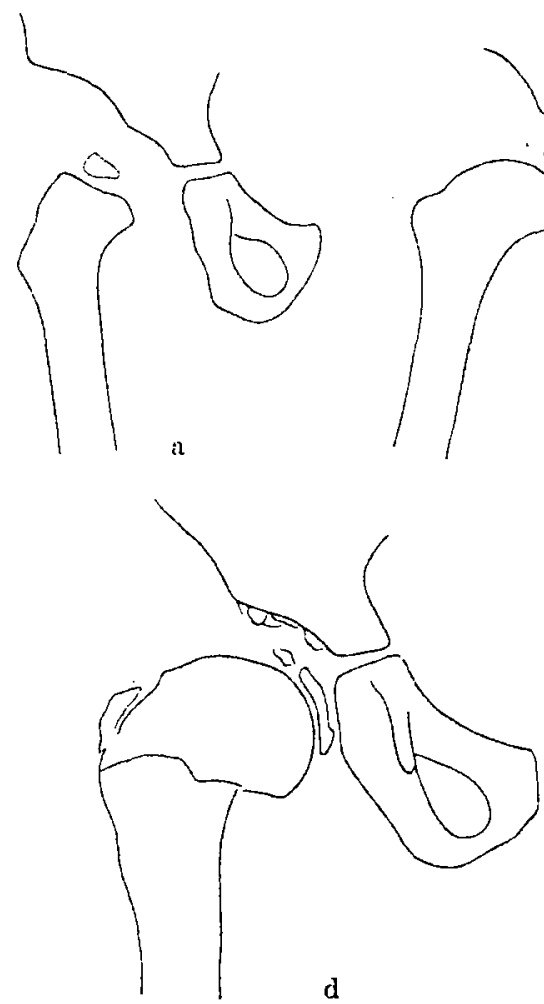

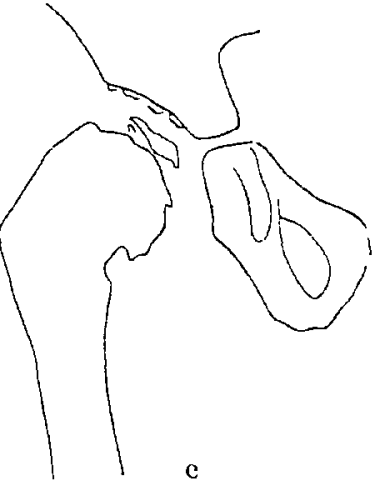

h
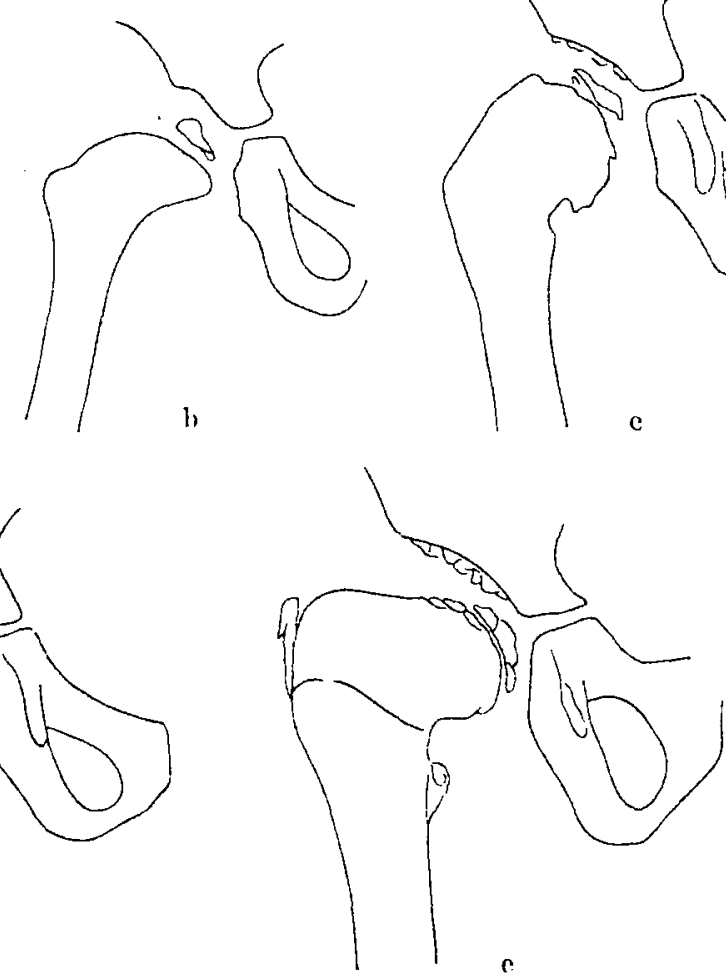

Ahb, 7. a vor der Reposition. b reponiert. c nach 2 Jahren. d nach 3 . Iahren. c nach 4 Jahren.

Im Gegensatz zur Kongenita scheint also hier der Krankheitsprozeß vorwiegend in der Kopfepiphyse zu sitzen und der Hals schon auf den gewöhnichen Wachstumsanreiz der normalen Belastung hin derartigen Umfang anzunehmen. Daß trotzdem eine Halsverbiegung (Abb. $7 \mathrm{~d}$ ) eintritt, ist einfach eine Folge des Mißverhältnisses zwischen Belastung und Tragkraft. Das stark wuchernde osteoide Gewobe, sowohl periostaler als auch enchondraler Herkunft wird so langsam verkalkt, daß der Hals biegsam bleibt. Auch hier liegt es zwar nahe, die primäre Ursache in einer Störung der Ossifikation (Puachitis) zu suchen, zumal am gesunden Bein ebenfalls gar nicht selten eine Coxa vara beobach tet worden ist. Nur hat man bisher gelernt, daß eine Rachitis nie lokal auftritt 
und demgemäß traumatische Beeinträchtigung der Wachstumszone, sowie rein mechanische Kräfte (Druck, Zug) zur Erklärung derartiger Schenkelhalsverbiegungen herangezogen. Nach den Frommeschen Untersuchungen bei der Spätrachitis sind wir jedoch gezwungen, mit dieser Anschauung zu brechen. Ist es ihm doch gelungen, gar nicht so selten rein lokale Störungen in den Wachstumszonen nachzuweisen. Das Verhalten der Trochanterepiphyse, die für ein 6 jähriges Mädchen doch sehr kümmerlich entwickelt ist, gibt hier recht wertvolle Hinweise. Diese Erscheinung nur als eine Folge der funktionellen Beanspruchung zu deuten, kann wenig befriedigen, vielmehr weist auch hier die unregelmäßige Form, das Zurückbleiben in der Größe auf eine Stōrung in der enchondralen Ossifikation hin, die dem Krankheitsbilde der Spätrachitis sehr nahe kommt. Erst dann sind die mechanischen Kräfte imstande, eine Deformierung herbeizuführen (Abb. 7e). Lange hat den un-

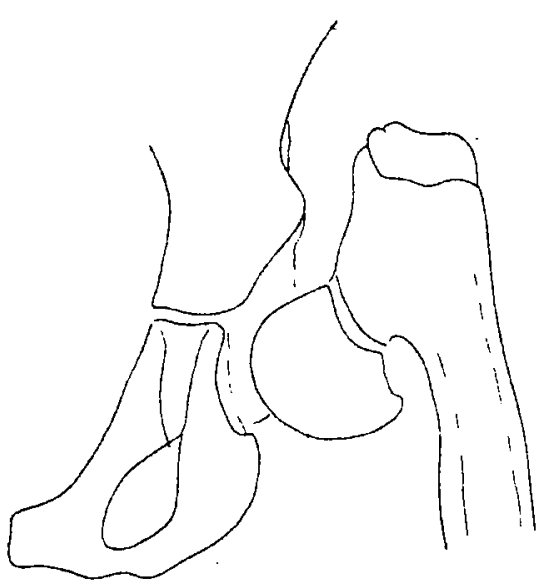

Abb. 8a. Coxa vara congenita. 131/2 jäh. riger Kinahe.

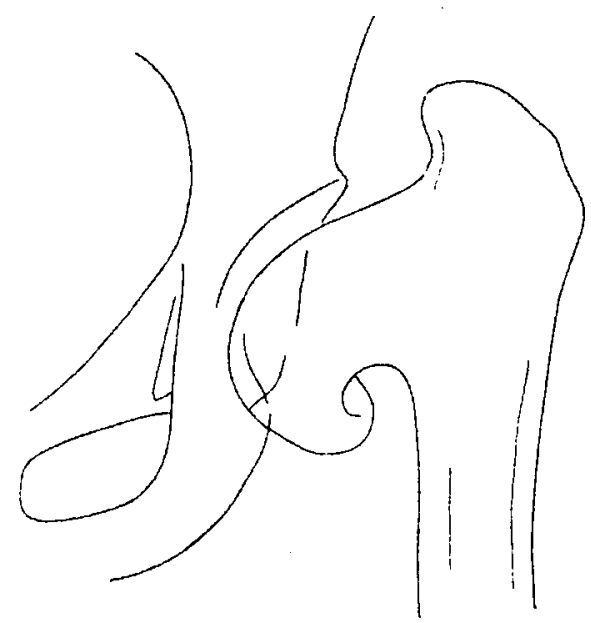

Abb. 8b. Spätform der Coxa vara nach kongenitaler Luxation.

regelmäßigen, mit Knorpelwülsten angefiilten Pfannengrund für die Kopfveränderungen bei der kongenitalen Hüftluxation verantwortlich gemacht. Eine pathologische Belastung allein kann derartige Veränderungen aber nicht bewirken. Es sind dazu osteomalazische oder rachitische Prozesse im Kuochen nötig, wenn nicht der Befund Preisers einen Fingerzeig bedeutet. Preiser fand neben dem Schwunde des Kopfkernes auch ein Zurückbleiben der Knochenkernentwicklung an der Hand und neigte zur Annahme einer allgemeinen Hypoplasie des Knochensystems, die sich jedoch sonst nur bei krankhafter Veränderung gewisser Drïsen mit innerer Sekretion (Thyreoidea, Hypophyse) vorfindet.

Die Prognose bei der Coxa vara congenita ist eine sehr ungünstige. Meist nehmen die Deformierungen $\mathrm{zu}$, so daß schließlich der Trochanter an der Beckenschaufel und der Kopf am Schenkelschaft stehen (Abb. 8a). Am Fehlen des Halses, am großen Kopf - eine Epiphysenlinie ist hier nicht vorhanden und an der bandartigen, von innen oben nach außen unten verlaufenden Aufhellung zwischen Kopf und Trochanter ist die angeborene Form unverkenubar. 
Auch bei den Schenkelhalsverbiegungen nach Luxationen sind die Heilungsaussichten gering, Wenn es nicht zu deformierenden Hüftgelenksveränderungen kommt, die schlieBlich zur Ankylose führen, so treten, falls nicht rechtzeitig entlastet wird, Folgezustände auf, die sich von der Kongenita nicht wesentlich unterscheiden (Abb. 8 b). Kopf, Hals und Rollhügel haben die Form eines Notenfähnchens angenommeu. Die Epiphysenlinie ist verknöchert.

Unter den postfötal erworbenen Leiden stellt die Rachitis die größte Zahl der Schenkelhalsverbiegungen. Von den 75 Fällen gehören allein zu dieser Gruppe 45. Dass Röntgenbild gibt natürlich je mach dem Grade und der Dauer, der Erkrankung ganz wechselvolle Befunde wieder. Bei einer floriden Rachitis kaun es zu sehr hochgradigen Halsverbiegungen kommen, so daß der Trochantes' hoch im Becken steht. Der Knochen zeigt wenig Kalkeinlagerung und fast gar keine Struktur. Die Epiphysenlinie ist erheblich verbreitert $(2-3 \mathrm{~mm})$, wolkig beschattet und verläuft schräg. Der Kopf ist deutlich marmoriert (Abb. 9a) und beginnt sich derart zum Halse cinzustellen, daß er mit seinem oberen Rande die verlängerte Schenkelhalsgrenze überragt und mit seinem unteren Saum die Halsmittelachse gerade erreicht. Durch die enorme Wucherung osteoiden Gewebes in der Knorpelwachstumszone und unterem Periost geht der Hals in die Breite und erhält so ein dickes, plumpes Aussehen, das außer bei der Rachitis nur ähnlich bei der kongenitalen Hüftluxation anftritt. In einem Zustande der Besserung wird die Knochenstruktur deutlicher, kräftiger, der Kopf kompakter. In den schweren Fällen bleibt aber eine exzentrische Stellung des Kopfes bestehen (Abb.9b), der erst nach längerer Einwirkung der normalen Körperlast wieder an seine Ausgangsstelle zurückgepreßt wird.

Ich stelle mir hierbei die Knorpelfuge infolge der mangelhaften Kalksalzeinlagerung nachgiebig vor, so da 3 die Schwerkraft des Körpers vermittels der Kopfkappe einen gleichmäßigen schiefen Druck auf die Epiphysenfuge ausübt und nach dem Parallelogramm der Krïfte in zwei anfeinander senkrecht stehende Komponenten zerlegt, schließlich die Kopfkappe in der Richtung der Epiphysenlinie nach abwärts treibt. In seiner Arbeit über die Spätrachitis in ihrem Zusammenhang mit den verschiedensten Krankheitsbildern (Bruns' Beitrïge 1920, Bd. 118) nimmt Fromme die Schwerkraft des Körpers für eine ganz ungleichmäßige Belistung der Wachstumszone zur Erklïrung der Schenkelhalsverbiegungen in Anspruch. Nach der beigefïgten Abbildung kinn ich mir schlecht rein mechanisch eine solche Wirkung vorstellen. Sie käme auch nur dann in Frage, wenn die Epiphysenlinie nicht schrïg von oben außen nach unten inner, sondern wie im Fickschen Lehrbuch (Anatomie der Gelenke) im oberen Teile horizontal verliefe, un von der Mitte an nach unten abzubiegen.

Umgekehrt steht die Bewegung des Halses nach unten entweder mit dem Druck auf den Trochanter (Seitenlage im Bett) oder mit dem dauernden Muskelzug der Glutaei im Zusammenhang. In einem weiteren Zustande der Besserung wird die Struktur immer klarer (Abb. 10). Die Epiphysenlinie ist noch $1-2 \mathrm{~mm}$ breit und verläuft wellenförmig. die untere mediale Halsspitze vogelschnabelartig umgrenzend. Bemerkenswert ist, daß der Trochanterepiphysenkern noch fehlt $\left(5 \frac{1}{2}\right.$ Jihre $)$. 


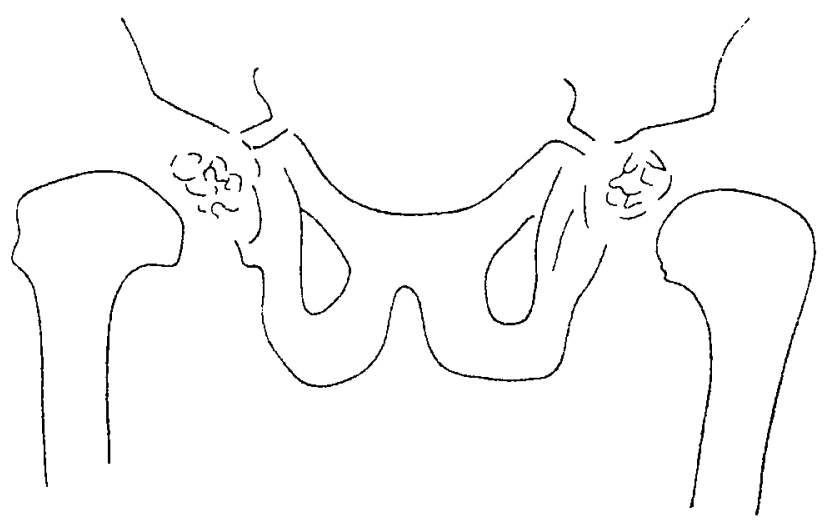

Abb. 9a. Rachitische Coxa vara.

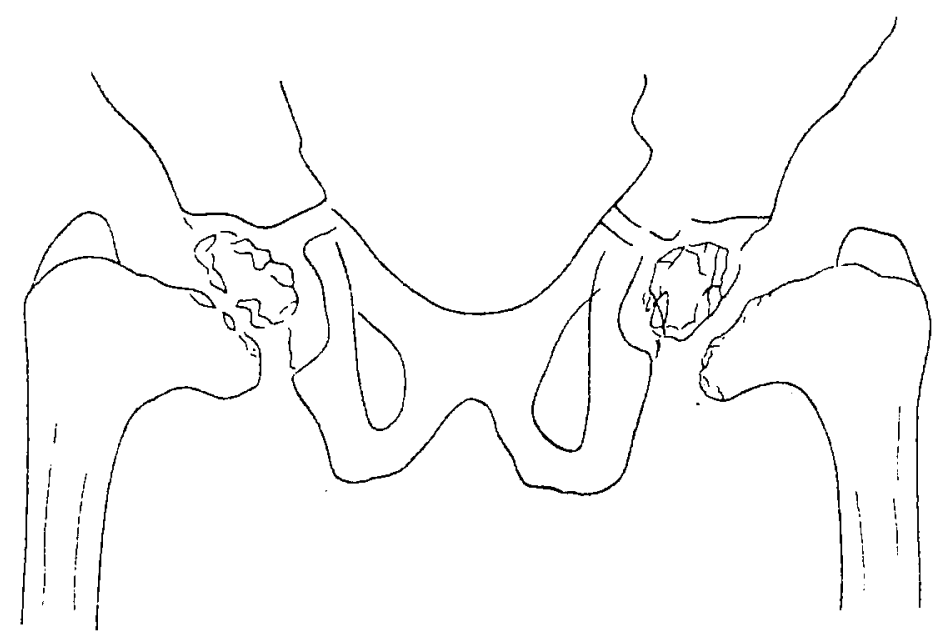

Abbleb. Rarhitische Coxa vara. 61/2 Jahre.

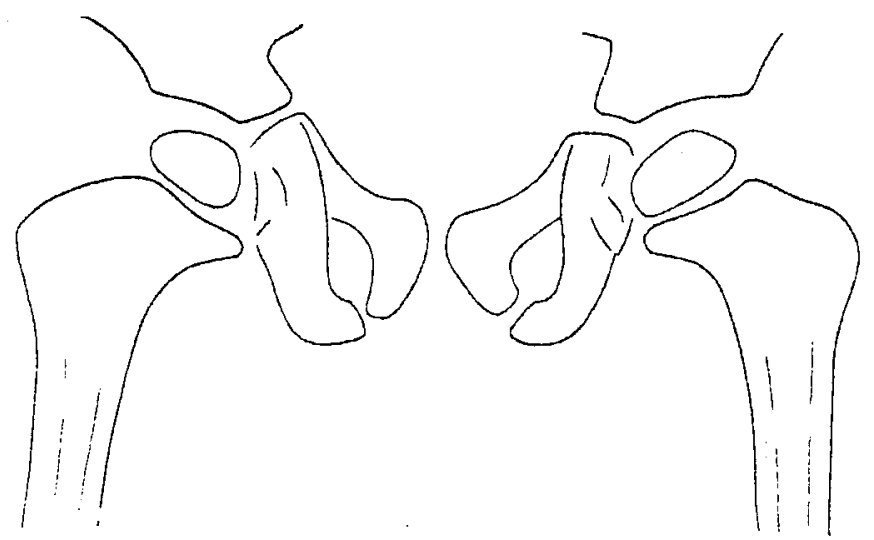

Abb. 10. $5 \frac{1}{2}$ Jahre alte Coxa vara rachitica. 
Fine Kopfforn, die ich öfters angetroffen habe, findet sich in Abh. 11 einer Coxa vara infolge rachitischer Verbiegung des Schenkelschaftes: Auch hier wellenförmige Epiphysenfuge, $1-1^{1 / 2} \mathrm{~mm}$ breit. Dieser wellige Verlauf der Knorpelfuge sowie die eigentümliche Kopfform sind immerhin auffallend und gar nicht so selten. Meistens ist die untere Halsspitze noch erheblich nach unten abgebogen. Wie kommen diese Formveränderungen zustande? Die eine Möglichkeit ist die, daß die im Zentrum liegenden Knochenlamellen von der Wachstumszone her der Belastung zuerst nachgeben, während der Zugbogen, besonders aber der Druckbogen, widerstandsfähiger bleiber. Auch dieser kann die Last auf die Dauer nicht allein tragen und biegt an seiner äubersten Spitze um. Der eigentümliche Kopf hat sich nur der Fpiphysenf uge und den veränderten mechanischen Verhä]tnissen angepabt. Der zweite Erklärungs-

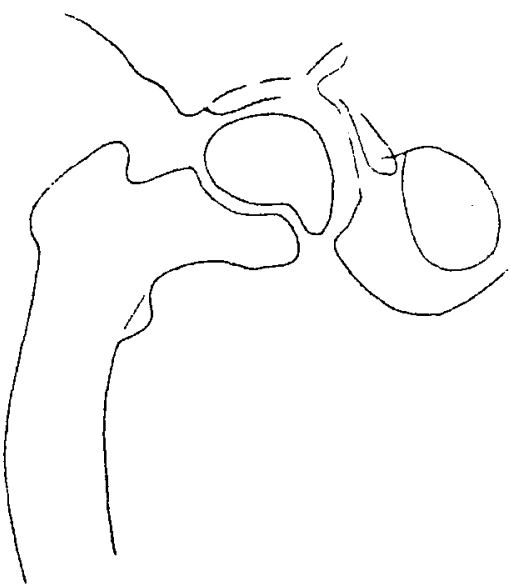

Abb. 11. Coxa vara rachitica. versuch liegt darin begrüindet, daß in leichten Fällen sowie am Begimn oder während der Ausheilung der Rachitis eine vermehrte Belastung einen Wachstumsreiz setzen kann im Sinne des Arndtschen biologischen Grundgesetzes, so daB am Druckbogensystem, welches die Hauptlast abfängt, sich größere Knochemmassen anlagern, bis die untere Halsspitze schnabelförmig vorspringt. Auf jeden Fall ist der Verlauf der Epiphysenfuge ein Produkt aus krankhaften Veriinderungen in der Verknöcherung und den Belastungsverhältnissen.

Als charakteristisch für die Coxa vara rachitica verdienen außerden hervorgehober zu werden die meist doppelseitige Verkleinerung des Schenkelhalsneigungswinkels und die rachitischen Verbiegungen an den Schenkelschäften. Der Schenkelhals ist im Gegensatz zur kongenitalen Coxa vara stets erhalten. Der Epiphysenwinkel hält sich zwischen 45 und 65 Grad.

Diese für die Coxa vara rachitica eigentümlichen hochgradigen Veränderungen des oberen Femurendes zeigen eine auffallende Neigung, sich zurückzubilden. Der Hals richtet sich wieder auf und verliert seine plumpe Gestalt. Dex Kopf rückt wieder in seine normale Laage zum Halse. Diese Tendenz ist bei der angeborenen Form nicht zu beobachten, und daher differentialdiagnostisch verwertbal. In floriden Stadium ist dagegen die Abgrenzung gegen die Coxa vara bei Tuberkulose schwer. Auch hier ist die Epiphysenfuge unscharf begrenzt, wolkig heschattet. Fleckige, scheckige Atrophie im angrenzenden Kopf und Hals, eventuell Kopfdefekte sind bei ihr zu finden. Da aber die Coxil vara rachitica fast nienals ohne Zeichen einer Allgemein-Rachitis auftritt, sind diese für die Diagnose ausschlaggebend.

Wit der Rachitis in innigem Zusammenhang stehen die beiden folgenden Abschnitte der Helbingschen Finteilung. Nach den neuesten Forschungen ïber die Spätrachitis bei Gelegenheit der Göttinger Epidemie 1919 durch From me ist es fast als sicher zu bezeichnen, diß sowohl ,die noch nicht sicher 
zu bestimmende Erkrankung des Wachstumsalters:" als auch die Osteonalazic Teilerscheinungen derselben sind. Allerdings sind die Röntgenbefunde $\mathrm{Abb}$. 12 und 13 gruudverschieden von denen der Frührachitis, so daß sich auf den ersten Blick kaum ein Zusammenhang erkennen läßt. Wenn man aber bedenkt, daß hier das fast in seiner Form vollendete Femurende, dort dagegen der Knochen in seiner stärksten Wachstumsperiode betroffen wird, so bietet die Erklärung des mechanischen Zustandekommens dieser hochgradigen statischen Verbiegungen kaum noch Schwierigkeiten. Der Krankheitsprozeß verläuft auch hier zunächst in der Wachstumszone des Schenkelhalses. Mit ihrer Verbreiterung und Lockerung geht Hand in Hand die wachsende Empfindlichkeit gegen Druck und Stoß. Schon die normale Körperlast und die' langandauernden Erschütte-

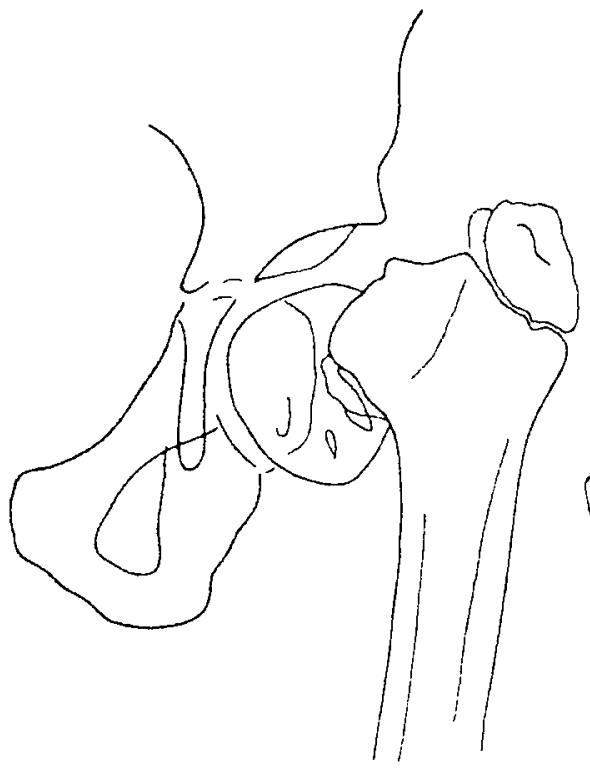

Abb. 12. Coxa vara statica. 12 Tahre.

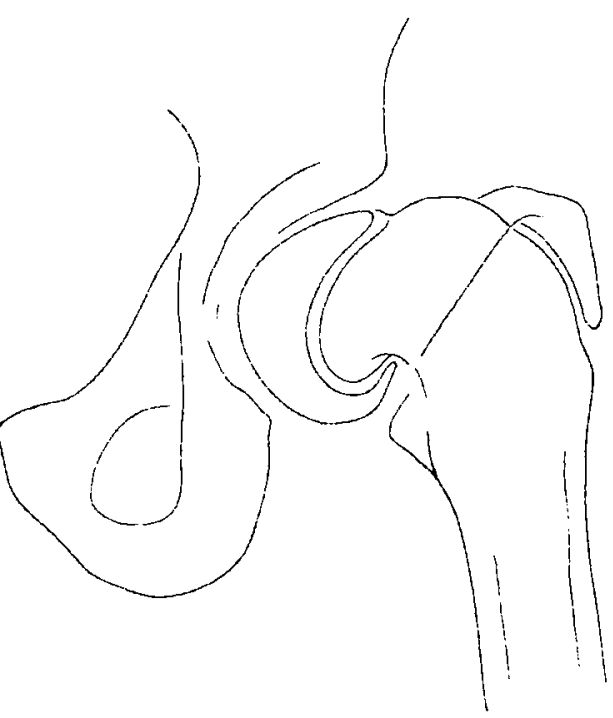

Ablu. 13. Coxa vara statica.

rungen beim Gehen und Laufen gemügen als auslösende Crsache für das $A b-$ rutschen des Kopfes. Soweit das Wachstum noch nicht abgeschlossen ist, wird auch das unvollständig verkalkte asteoide Gewebe Druck- und Zugveränderungen unterliegen. Dabei mag es dahingestellt seim, ob die Kopfepiphyse auch von der Knorpelfuge, vom Rande des Knochenkerns oder dem Gelenkbnorpel. der in der Adoleszenz mit dem Knochenkernrande zusammenfällt, wächst. Bei der Coxa vara statica oder adolescentium sind aber die Unterschiede gegenüber dem Normalen zu hochgradig, als daB sie allein in der Erkrankung der Knorpelfuge und Ungestaltung des wachsenden Knochens ihre Erklärung fänden. Vielmehr muß man einen Kalksalzschwund im fertigen Knochen der ganzen Kopfepiphyse und des angrenzenden Halsteiles annehmen, so daß auch diese Teile Druckveränderungen unterliegen. Sehr schön ist in Abb. 13 zu sehen, wie die weiche Kopfkappe gegenüber dem unteren Pfanneurand machgegeben hat und eine deutliche Eindellung aufweist. Der Kopf ist 
infolge der dauernd wirkenden Druck- und Zugkräfte abgeplattet und lang ausgezogen (Mondsichel). Nur $2 / 3$ der Kopfkappe stehen noch in der Pfanne, $1 / 3$ schaut unten heraus, die Epiphysenlinie neigt sich im oberen Teile sehr der Senkrechten zu und biegt im unteren ziemlich scharf nach außen ab, wie ein Komma. Infolge der hochgradigen Umknickung des Halses nach hinten (Retroversion) könnte man geneigt sein. einen Teil dieser Gestaltsveränderungen auf die Projektion zurückzuführen. Die Resektionspräparate Müllers, Hoffmeisters und auch Frangenheims bestätigen jedoch den Röntgenbefund vollauf.

Ähnliche Bilder wie bei der Statika können auch bei der rein traumatischen Coxa vara vorkommen, etwa nach einem Schenkelhalsbruch oder nach einer direkten Epiphysenlösung. Wie bei jeder Fraktur laufen hierbei Knochenaufund -Abbau nebeneinander her und schaffen eine gegen Zug und Druck nachgiebige Verkittung der Bruchflïchen, wenn diese ïberhaupt in normaler Stellung zueinander liegen. Die Epiphrsenlinie weist bei der Coxa vara traumatica somit keine bestimmten GesetzmäBigkeiten anf.

Fälle von Osteomyelitis, Tumoren, Ostitis fibrosa mit Coxa vara wurden nicht beobachtet. Sie bieten auch nach den Aufzeichnungen in der Literatu: nichts Charakteristisches an der Epiphysenfuge, da vorwiegend der Scheakelhals von diesen Erkrankungen betroffen wird. Die Prognose der statischen und traumatischen Coxa vara richtet sich nach der Schwere der Deformitit, Rückbildungen wie bei der Rachitis werden nicht beschrieben. Durch eine rechtzeitige Entlastung läßt sich das Fortschreiten der Deformierung zwar hintanhilten, leider wird aber das furktionelle Ergebnis oft dadurch getrübt, daß sich Gelenkveränderungen im Sime der Arthritis deformans einstellen. deren auslösende Ursache wohl nach Preiser in der Inkongruenz der Gelenkflächen von Kopf und Pfanne zu suchen ist.

\section{Zusammenfassung.}

Fïr die Coxit vara congenita lassen sish nux mit Vorbehalt aus dem Röntgenbilde Gesetzmäßigkeiten herauslesen, da sich Veränderungen ähnlicher Art wuch bei der Rachitis finden, ja, es demnach nicht sehr fern liegt, die Kongenita iiberhaupt als eine besondere Form der Richitis zu betrachten.

Bei der Coxa vara nach kongenitaler Luxation spielen sich dic schwersten Veränderungen im Kopf ab, die sehr an das Bild der Osteochondritis (Perthes) erimern. Die Verbiegungen im Hialse sind auf osteomalazische Prozesse zurückzuführen.

Die rachitischen Schenkelhalsverbiegungen bieten soviel feststehende Kenmzeichen, daß höchstens im floriden Stadium eine Verwechselung mit der Tuberkulose möglich ist.

Auch für die Statiki werden osteomalazische oder rachitische Prozesse verantwortlich gemacht.

Die Befunde wurden an 75 Coxa vara-Fällen erhoben, von denen allein 45 auf eine einwandfreie Rachitis im Kindesaltel von 2-3 . Tahren zurückzufïhren waren.

Das Verhalten der Epiphysenlinie (Verlauf, Form, frühzeitiges Verschwinden oder längeres Sichtbarbleiben) ist ein Produkt aus dem Ablauf der Verknöcherung und den Belastungsverhältnissen. 